\title{
Letter to Editor \\ Coronavirus Pandemic a Factor in Delayed Mourning in Survivors: A Letter to The Editor
}

\author{
Seyyed Mohammad Hossein Javadi ${ }^{1}$ (), *Maryam Sajadian² ${ }^{2}$
}

1. Department of Social Welfare, University of Welfare and Rehabilitation Sciences, Tehran, Iran.

2. Department of Counseling, University of Welfare and Rehabilitation Sciences, Tehran, Iran.

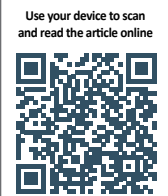

Citat ion: Javadi SMH, Sajadian M. [Coronavirus Pandemic a Factor in Delayed Mourning in Survivors: A Letter to The Editor (Persian)]. Journal of Arak University of Medical Sciences (JAMS). 2020; 23(1):2-7. https://doi.org/10.32598/JAMS.23.1.4578.3

doil https://doi.org/10.32598/JAMS.23.1.4578.3

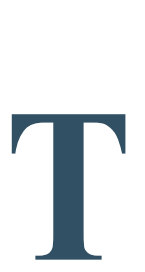

\section{Dear Editor}

he coronavirus newness and its high rate of spread have feared most people, a fear that in many cases causes social and psychological problems. Common psychological reactions include COVID phobia, anxiety, depression, sleep disturbances, obsessive-compulsive disorder, and post-traumatic stress disorder - just some of the associated problems related to coronavirus. Other problems caused by the disease include home quarantine, disruption of daily activities, obsession with cleanliness and washing, abnormal mourning for the death of loved ones, death of people, lack of equipped hospitals, difficulty in performing hospital duties, the fatigue of medical staff, illness and shortages in medical staff, hypersensitivity in some occupations due to disease control and treatment, economic problems of the community, closure of schools, universities, and offices, rumors in cyberspace and society [3].

Coronavirus has spread to many countries around the world and has claimed many lives. As of today, April 28, 2020, reports from the Ministry of Health, Treatment and Medical Education show that 92584 people have been infected in Iran, of whom, 5877 have died. According to the report, the number of people with coronavirus in the world is 3106000 , of whom, more than 200000 have died. Unfortunately, the virus is not the only cause of death. Another important issue is the family of these people, who are not allowed to mourn, vent their emotions, and express grief.
They get quarantined for a long time after the death of a loved one and cannot communicate with other relatives.

Also, the family of these people may be mentally ill when they have to mourn, but they can't mourn, or they may suffer from mental rumination during the quarantine period. One of the family members said, "I can't believe my spouse died easily. He was only 35 years old and had no illness. My wife was very kind. She died in the worst possible way. She died while she was very oppressed. There was no mourning ceremony or anyone". He expressed his condolences. "Sometimes I get angry with my relatives for not coming to my senses, and then I say no, I don't have the right to come". Besides, the person may experience delayed mourning at least 6 months later due to these conditions [4].

Delayed mourning is the lack of timely mourning or timely restraint; in fact, in these cases, the person may overreact, i.e., mourning, in the future due to a lack of proper emotional response. The main feature of late mourning is the long-term denial of the issue of loss. In other words, when these people lose loved ones, those around them and society prevent them from grief, sadness, and anxiety and do not allow them to mourn. They develop delayed mourning. Delayed grief causes psychological problems such as depression, anxiety, bipolar disorder, obsessive-compulsive disorder, sleep disturbances, eating disorders, anger, guilt, suicide, drug addiction, and so on [5]. It is recommended that virtual mourning ceremonies be held for these people instead of traditional mourning ceremonies. In the experi-

\section{* Corresponding Author:}

Maryam Sajadian, PhD.

Address: Department of Counseling, University of Welfare and Rehabilitation Sciences, Tehran, Iran.

Tel: +98 (918) 3408562

E-mail: maryamsajadian1990@gmail 
ence of mourning in cyberspace, it is possible to share audio, text, photos, and videos, and this type of mourning is beyond geographical boundaries [6].

We can propose protocols for this issue as the research findings indicate the relationship between delayed mourning and mental disorders [7]. In this regard, the need to design and develop virtual and online mourning for the family of these people is felt. Online access such as social networks, the Internet, telephone, and media can be a good platform to present this protocol. The target population of this protocol is the family of people whose loved one died of coronavirus. In general, considering the emphasis of the World Health Organization and the Ministry of Health, Treatment and Medical Education on the access of all people to health information [8], it is necessary to provide a suitable platform for the fulfillment of this mission.

Therefore, preparation, organization, and making accessible health information available at the right time and place to everyone helps reduce the cost of health care [9], disease prevention, and community health promotion. Finally, it is necessary for the government to anticipate these rights in national health information policies and to provide the necessary basis for their implementation.

\section{Ethical Considerations}

\section{Compliance with ethical guidelines}

All methods used in the study of human participants are in accordance with the criteria of the Ethics Committee.

\section{Funding}

This article is a letter to the editor and has not received any financial support from the government or academic organizations.

\section{Authors' contributions}

Study Design, data collection, writing the manuscript: Maryam Sajadian; Providing the scientific data, revising the material for the final version: Mohammad Hossein Javadi; Approving the final manuscript: All authors.

\section{Conflicts of interest}

The authors declared no conflict of interest.

\section{Acknowledgements}

The authors would like to thank the authorities of the University of Social Welfare and Rehabilitation Sciences. 
This Page Intentionally Left Blank 


\title{
ياندمى كروناويروس عاملى براى سوتى تأخيرى در بازماندَّان: نامه به سردبير
}

\author{
"سيد محمد حسين جوادى' هـ، مريم سجاديان' \\ ا. كروه مددكارى اجتماعى، دانشكاه علوم بهزيستى و توانبخشى، تهران، ايران. \\ r. كروه مشاوره، دانشعاه علوم بهزيستى و توانبخشى، تهران، ايران.
}

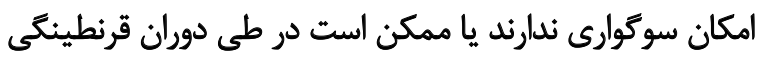

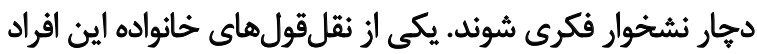

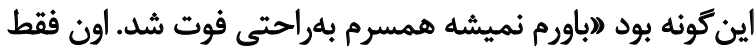

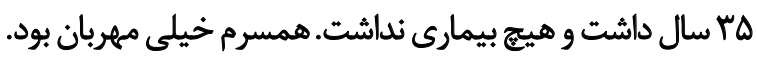

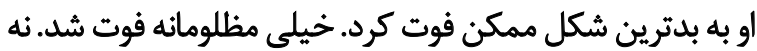

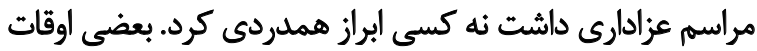

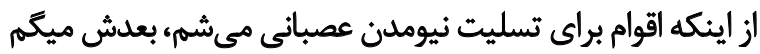

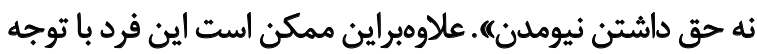

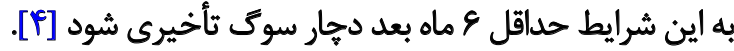
سوك تأخيرى عبارت است از عدم سوكوارى به موقع يا مهار

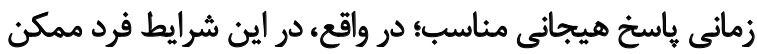

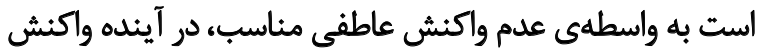

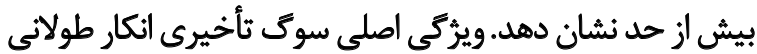

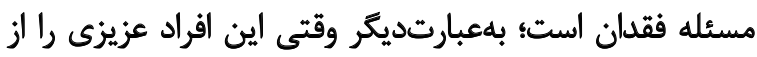

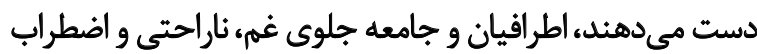

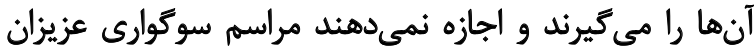

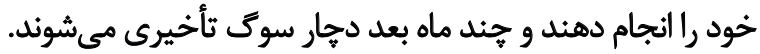

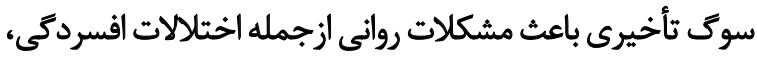

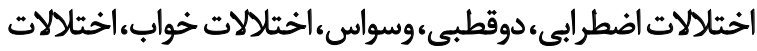

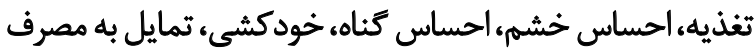

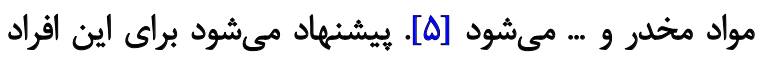

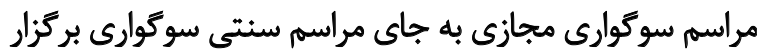

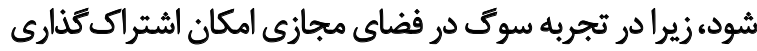

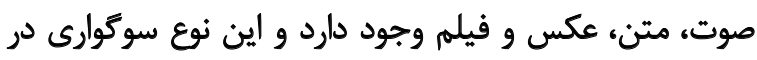

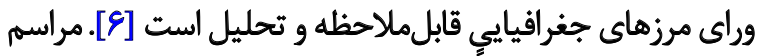

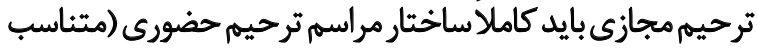

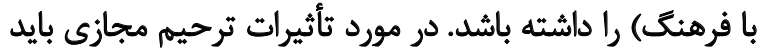

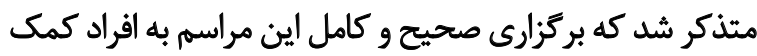

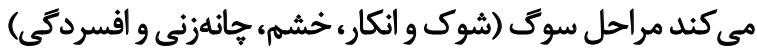

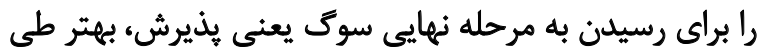

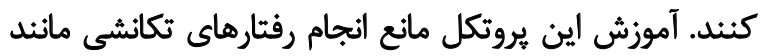

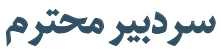

جديد بودن كروناويروس و سرعت بالاى ابتلا به آن باعث شده

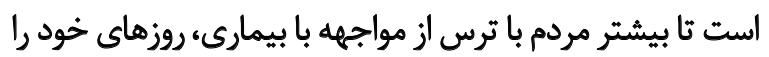

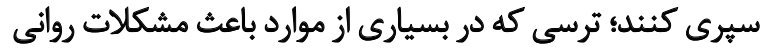

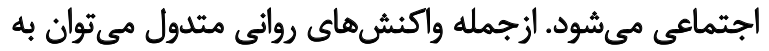

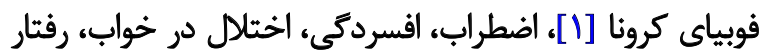

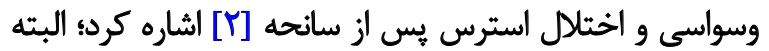

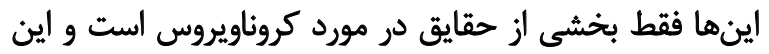

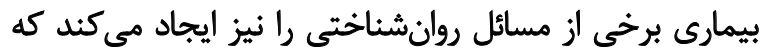

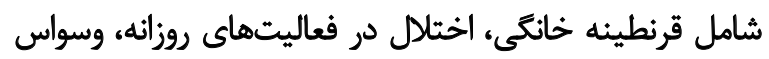

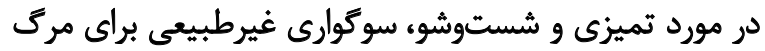

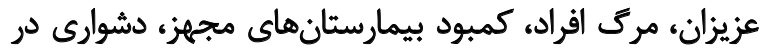

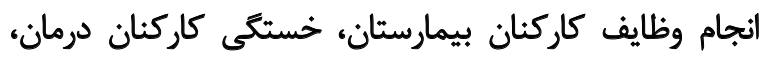

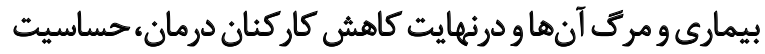

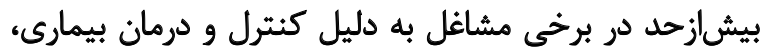

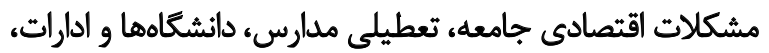

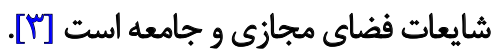

كروناويروس در بسيارى از كشورهاى جهان شيوع ييدا

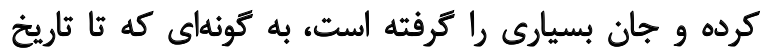

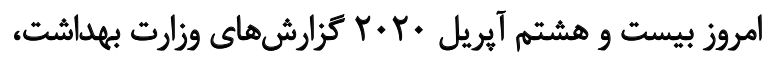

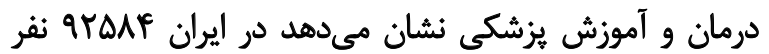

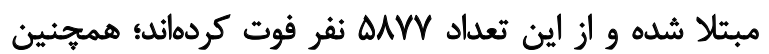

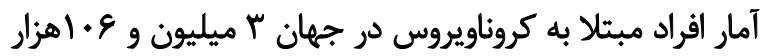

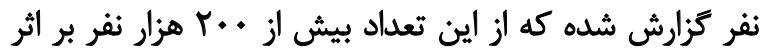

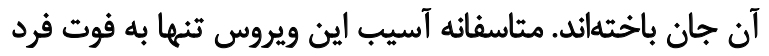

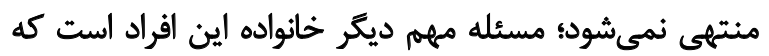

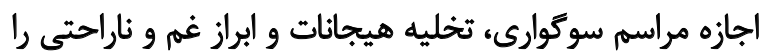

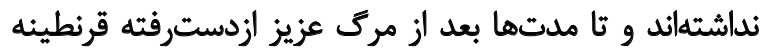

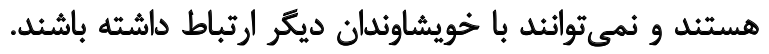
همجنين خانواده اين افراد در زمانى كه بايد سوكوارى كنيند، 


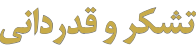

نويسندًان از دانشًاه علوم بهزيستى و توانبخشى كمال تشكر

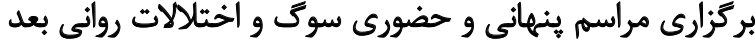

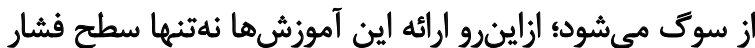

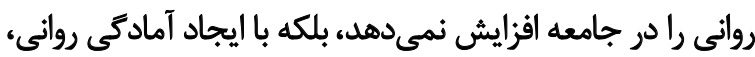

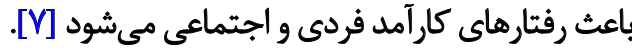
ما مىتوانيم براي اين مسئله بروتكلهايي در نظر بكيريم.

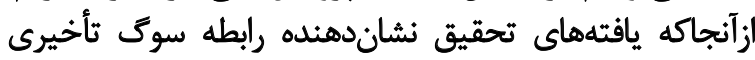

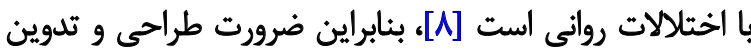

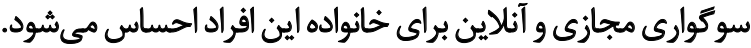

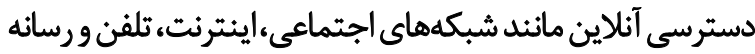

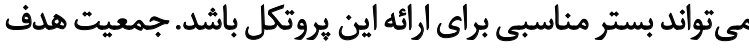

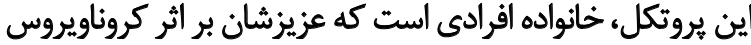

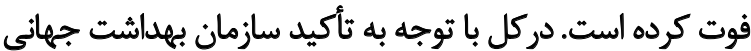

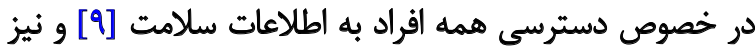

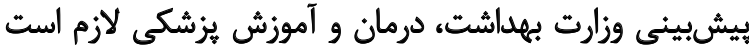

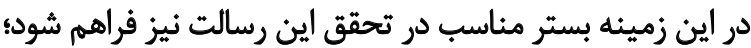

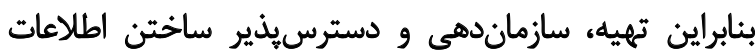

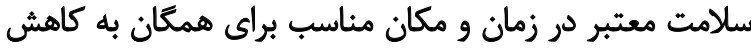

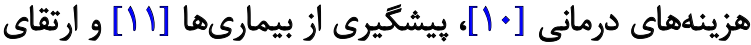

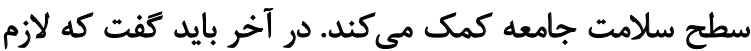

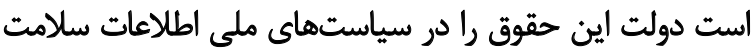
ييش بينى كند و بستر لازم را براى اجراي آن فراست فراهم آورد.

مالاحظات اخلاقى

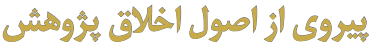

كليه شيوههاى انجامشده در مطالعه در مورد شركت كنيدكان انسانى مطابق با معيارهاى كميته اخلاق است.

$$
\text { ماهم }
$$

اين مقاله يك نامه به سردبير است و هيج حمايت مالى از سازمانهاي دولتى و دانشكاهى دريافت نكرده است.

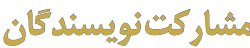

مريم سجاديان طراحى مطالعه، جمعآورى دادهها و تهيه

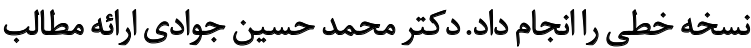

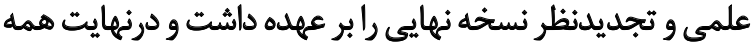
نويسندكان نسخه نهايى را مطالعه و تصويب كردند.

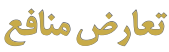

نويسندكان بيان كردئد كه هيجّونه تعارضى در منافع وجود 


\section{References}

[1] Zarghami M. Psychiatric aspects of Coronavirus (2019-nCoV) infection. Iran Psychiatry Behave Sci. 2020; 14(1):e102957. [DOI:10.5812/ ijpbs.102957]

[2] Huang $Y$, Zhao N. Generalized anxiety disorder, depressive symptoms and sleep quality during COVID-19 epidemic in China: A webbased cross-sectional survey. Psychiatry Res. 2020; 288:112954. [DOI:10.21203/rs.3.rs-17172/v1] [PMID] [PMCID]

[3] Javadi MH, Arian M, Qorbani-Vanajemi M. The need for psychosocial interventions to manage the Coronavirus crisis. Iran Psychiatry Behave Sci. 2020; 14(1):e102546. https://sites.kowsarpub.com/ijpbs/articles/102546.htm

[4] Shear MK. Complicated grief. N Engl J Med. 2015; 372(2):153-60. [DOI:10.1056/NEJMcp1315618] [PMID]

[5] Sadock B, Sadock VA, Sussman N. Kaplan \& Sadock's pocket handbook of psychiatric drug treatment. Philadelphia: Lippincott Williams \& Wilkins; 2017. https://books.google.com/books?id=Z_tNDwAAQBAJ\&dq

[6] Kalan M. Expressions of grief on facebook: Navigating discomfort, persistent identity, and public memorialization [MA. Thesis]. New York: Syracuse University; 2013. https://surface.syr.edu/thesis/18/

[7] Gruenwald H, Gruenwald L. Cyber cemeteries and virtual memorials: virtual living monuments as on-line outlets for real life mourning and a celebration of life. In: Cox GR, Bendiksen R, Stevenson RJ. Making sense of death: Spiritual, pastoral, and personal aspects of death, dying, and bereavement. Amityville: Baywood Publishing Company; 2003. https:// books.google.com/books?id=aChHAAAAMAAJ\&q

[8] Luber M. Protocol for excessive grief. J EMDR Pract Res . 2012; 6(3):12935. [DOI:10.1891/1933-3196.6.3.129]

[9] Aminpour F. Can we achieve health information for all by 2015. Info Manag Health. 2005; 2(1):64-72. https://vlibrary.emro.who.int/imemr/ can-we-achieve-health-information-for-all-by-2015/

[10] Zeinali V, Riahinia N. Information therapy services in educational hospitals: a feasibility study in educational hospitals of Shahid Beheshti University of Medical Sciences. J Health Adm. 2015; 18(59):66-81. http:// jha.iums.ac.ir/article-1-1640-en.html

[11] Killeen MB, Barnfather JS. A successful teaching strategy for applying evidence-based practice. Nurse Educator. 2005; 30(3):127-32. [DOI:10.1097/00006223-200505000-00016] [PMID] 